\title{
The Study Edge Detection of Medical Images using Transformation Techniques and Filteration Methods
}

\author{
Harneet Kaur \\ M.Tech (student) \\ Baba Banda Singh Bahadur Engineering College, \\ Fatehgarh sahib, Punjab, India
}

\author{
Ishpreet Singh \\ Assistant Professor \\ Baba Banda Singh Bahadur Engineering College, \\ Fatehgarh sahib, Punjab, India
}

\begin{abstract}
Edge is an essential characteristic of an image. Edges can be defined as boundary between two different regions in an image. Edge detection refers to the progression of identify and locate sharp discontinuities in an image. Edge detection processes considerably reduce the quantity of data and filters out useless information, while preserving the essential structural property in an image. Because computer apparition involves the recognition and classification of objects in an image, edge detections is a vital tool. Edge is a basic and important feature of an image. Image is a combination of edges. Detecting edges is one of the mainly significant features in image segmentation. Edge detection is a vital step as it is a process of identifying and locates sharp discontinuities in a representation. In this paper, the main intend is to swot edge detection process based on different techniques and most commonly used edge detection techniques such as Sobel, Prewitt, Roberts, Canny, and Laplacian Gaussian.
\end{abstract}

\section{Keywords}

Edge Detection, Filters, Process of detection process, canny and Sobel techniques.

\section{INTRODUCTION}

Image dispensation systems to understand an image, the separation of the picture into thing and background are a critical step. Segmentation divider the image into a set of displace region that are visually different, consistent and meaningful with deference to some description or computed properties, such as grey level, intensity, texture or colour to allow easy image examination. An enormous number of methods are available in the creative writing to segment imagery. This is a crucial work since the production of an image segmentation algorithm can be feed as input to higherlevel processing tasks [1].

In day to day existence many such cases occur in which miniature hair line rupture may get ignored in the X-ray by the Doctors in such cases Bone fracture detection using image processing will help the doctor to avoid such errors. Digital image dispensation is an increasing area with application concerning to our daily lives, especially in progressive transmission of imagery video code, digital libraries image database, remote sensing, and other image database, remote sensing, and other and analysis techniques have been developed to aid the analysis of remote sensing images and to extract as much information as possible from the image [2]. The vast compilation of digital images are collected due to the development in the digital storage space media, image capture devices like scanners, web cameras, digital cameras and rapid growth in internet. These lead to fast and efficient repossession of these descriptions for visual information in different field of life like check-up, medicine, art, architecture, education, crime preventions, etc.

Edge based method is most usually used method to execute image segmentation. An edging may be regard as boundary between two unlike regions in an image [3]. The edges for an image are the important characteristics that put presumptuous an indication for a superior incidence. Edge detection is a terminology in image processing and processor hallucination, mainly in field of attribute detection and attributes extraction that plays an imperative role in segmentation of an imaginary for identification of matter. The development of detect edges for an image may make possible in image segmentation, data compression, and also help for image reconstruction.

Edge detection is a procedure in which the points where image brilliance changes brusquely or properly are identified. These points are organized underneath line segments called edges. Edge detection also aims to classify and place discontinuities in an image [4]. Noise and image both have high incidence, hence edge detection becomes hard. The main object of study a variety of edge detection technique and studying their performance is due to trouble such as false edge uncovering, noisy images, missing edges etc.

\section{DETECTION TECHNIQUES}

The points at which image intensity changes roughly are normally organized into a set of curled line segments termed edges. The edges extracted from a two-dimensional image of a three-dimensional [5] scene can be classified as either standpoint dependent or point of view self-governing. A point of view independent edge typically reflects inherent property of the three-dimensional objects, such as exterior markings and outside shape .A viewpoint charge edge may change as the standpoint changes, and characteristically reflect the geometry of the scene, such as objects occluding one another. A typical edge might for instance be the border flanked by a chunk of red colour and a wedge of yellow. In contrast a line can be a small number of pixels of a dissimilar colour on or else unchanging backdrop. For a line, there may therefore typically be one edge on each side of the line.

To the number of boundaries [6] in an image. There is an exceptionally large number of edge detection operators available, each of which are intended to be sensitive to certain edge types. Some variables that are involved in the collection of an edge uncovering operator include:

\subsection{Edge orientation}

The geometry of the operative determines a quality direction where it is most perceptive to edges. Operators can be reduced to seek horizontal, vertical, or diagonal edges. 


\subsection{Noise environment}

Edge detection is multipart in noisy images as both the noise and the edges have high-frequency satisfied. Operators used on noisy images are usually of larger range, so they can average sufficient data to reduction confined to a small area noisy pixels. This results in not as much of accurate localization of the detected edges.

\subsection{Edge structure}

All edges may not engage a step change in concentration. Effects like refraction or poor focus can end consequence in substance with boundaries defined by a steady change in intensity. The worker needs to be preferred to be receptive to such a slow change in those bags. In order to distinguish newer wavelet-based techniques actually exemplify the nature of the transition for each edge [7].

\section{Steps of edge Detection}

a) Filtration: Every image is associated with some intensity values, random change in these values can consequence in noise. Some ordinary noise is: salt and pepper noise, impulse noise etc. Noise can result in difficulties in effectual edge detection; therefore image has to be filtered in order to reduce the noise comfortable that lead to loss of edge strength [2]. It is also termed as Smoothening [8].

b) Enhancement: Improving the quality of image is termed as improvement. It aims to manufacture an image which is better and more suitable than original. A filter is applied in order to improve the quality of perimeter in image.

c) Discovery: Several methods are adopted to determine which points is edge point and which edge pixels have to be discarded as noise.

These steps should be followed carefully in order to detect the limits successfully, as the next steps are solely dependent on edges detected.

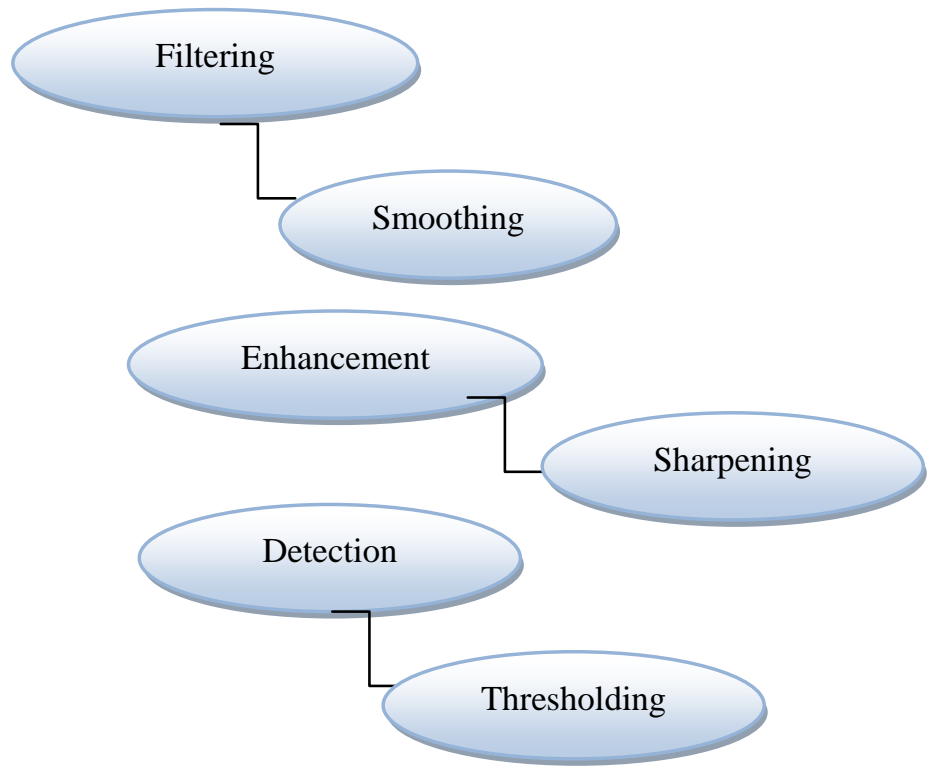

Figure 1: Steps of Edge Detection

\section{EDGE DETECTION IN MEDICAL FIELD}

The Edges in a digital image provide significant information concerning the objects contained surrounded by the image because they constitute boundaries between objects in the image. Images are prone to artefacts and noise. Salt \&intersperse noise is an appearance of noise characteristically seen on images. It is classically manifested as randomly occurring white and black pixels. Salt \&sprinkle noise creep into images in situations where quick transients, such as faulty switch. Otherwise, White noise is preservative in nature where the all pixel in the image is modified via the addition of a value strained from a Gaussian contribution. To exam the generalization of the results, the suggested edge detection algorithm was tested on medical imagery containing these type of noise . By health check image dispensation, researchers and clinicians can easily analysis the image data, thereby enhancing their ability to study, monitor, diagnose and treat check-up disorders. Medical image processing is currently finding wide request in a large variety laboratory research and scientific practice. Biologists learn cells and generate 3-D[9] co focal microscopy data sets; virologists generate 3-D reconstruction of virus from micrographs; radiologists recognize and count tumours from MRI and CT scans; and neuroscientists detect local metabolic brain activity from functional MRI scans and PET. Analysis of these diverse image types requires complicated automated quantification and visualization tools.

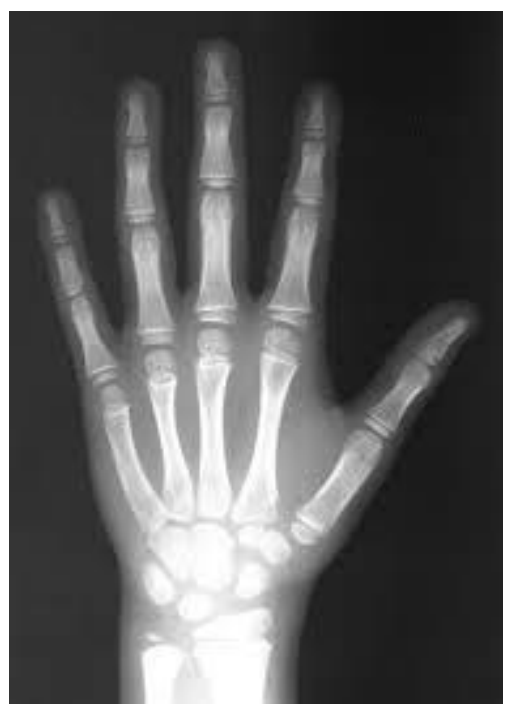

Figure 2 : X-Ray image of Bones

\section{RELATED WORK}

Julian et al [10] presented a method for vessel segmentation and track in ultrasound images using Kalman filters. tailored Star-Kalman method is used to determine vessel contours and ellipse parameters using an extensive Kalman filter with an indirect model. A temporal Kalman filter is used for tracking the vessel center over some frames, using place size from a handheld sensitized ultrasound probe. Segmentation and tracking is implement in real-time and validate using replicated ultrasound data with known features and real data. Results indicate that the signify errors between segmented contours and expert tracings are 1 to 2 percent of the maximum feature dimension. Francois et al [11] obtainable segmentation of intima-media thickness of carotid arteries in 
view of computing various dynamical property of that tissue, such as suppleness distribution. The echogenicity of a province of interest comprising the intimae-media layers, the lumen, and the adventitia in an ultrasonic B-mode image is model by a mixture of three Nakagami distributions. The means is well suited for semi-automatic context that requires minimal manual initialization. Juin et al [12] proposed segmentation for brain MR images using a dispersed transformation. This means extends the Gaussian mixtures expectation maximization segmentation to a power transformed account of mixed intensity distribution. Different correcting bias is applied previous to image segmentation to avoid performance degradation caused by intensity in homogeneity. The partition of brain tissues were validated and evaluated against physical segmentation from the internet brain segmentation repository and simulated image volumes from BrainWeb. Jiantao et al [13]urbanized an automatic method using computational geometry to notice and segment fissuredepict on Computed Tomography (CT) images. After geometric modelling of lung using march cube algorithm, Laplacian smoothing is functional to enhance pulmonary fissures by depressing nonissue structures while smooth the surface of lung fissure. An extended Gaussian image means is used to locate the fissures in arithmetical manner. The presentation was evaluated by thoracic radiologists using a set of 100 images randomly selected from 10 screening CT examinations. Veeralakshmi et al [14] proposed an edge detection technique for detecting the correct border of objects in an picture. The means can detect the boundaries of object using the information from intensity gradient using the vector image replica and feel gradient using the edge map model. Padmapriya et al [15] proposed a method to sense boundary in check-up image using size, direction, edge map and density of the edges bounded to the article and to crop the detect object and expand the image. The blur end product of the enlarged image is removed and a high decision image is bent from the low resolution image.

\section{PROBLEM FORMULATION}

$\mathrm{X}$-ray medical imaging plays an essential role in diagnosis of bone rupture in human body. The X-ray image helps the medical practitioners in choice making and effectual management of injury. In order to improve diagnosis results, the stored digital images are further analysed using health check image processing. The most common ailment of the human bone is fracture. Bone fractures are not anything but the crack which occurs due to accidents. There are many types of bone fracture such as normal, slanting, comminute, oblique, twisting, segmented, avulsed, impacted, torus and greenstick. Generally for X-ray image segmentation of bone fracture, a number of edge discovery algorithms like Sobel, pewit, Roberts and canny were used.

\section{EDGE DETECTION TECHNIQUES}

The edge discovery algorithms can be generally classified based on the behavioural study of edges with respect to the operator. Dissimilar edge-detection approaches can be broadly classified under Classical or Gradient based edge detectors (first derivative), Zero crossing [16] (second derivative) and Optimal edge-detector.

\section{a) First Order Derivative Based Edge Detection} Its detect the limits by seeking the maximum and minimum in the first derivative of the image. Sharpen an image offer grades in the detection of fine details and also in enhancing the blurred ones. The degree of the slope is the most influential method that forms the basis for a variety of approaches to sharpening. The incline vector points in the course of maximum rate of change.

\section{b) Laplacian based Edge Detection}

To find the edges, Laplacian method hunts for zero crossings in the moment derivative of the image. An edge has the 1-D shape of a slope and its place can be tainted by calculating the derivative of the image.

\section{c) The Roberts Detection}

The Roberts Cross operative performs a straightforward, rapid to compute, 2-D spatial gradient measurement on an image. Pixel values at each point in the production symbolize the estimated absolute magnitude of the spatial gradient of the input image at that point. The worker is complete up of a $2 \times 2$ convolution kernel.

\section{d) Discrete Wavelet Transformation}

The wavelet transform has grown pervasively approval in demising[17] of image as well as signal processing. It is the breaking down a definite signal into scaled along with shift versions of the unique wavelet. A wavelet is a type of waveform of efficiently restricted duration which has average value of zero. And for signals; the identity of the specific signal is specified through the component of low-frequency. The content of high-frequency only communicates nuance. If components of high frequency are extracted, the image which sounds different, but still it could be understood easily. If components of low frequency are extracted, the signal sounds will gabble. On implementing wavelet transformations on specific signal, detail and approximation components could be attained easily.

\section{e) Canny Operator}

1. It is a method to find edges by isolating noise from the picture without moving the features of the edges in the image and then applying the tendency to find the limits and the decisive value for threshold. The canny edge detector first smoothens the image to eliminate noise. Then it finds the image incline to emphasize region with high spatial derivatives. After that it performs tracking along these region and suppress any pixel that is not at the maximum. The gradient array at this moment can further be summary by hysteresis which is used to track along the remaining pixels that have not been suppressed. Hysteresis uses two threshold and if the extent is below the first threshold, it is set to zero. If the magnitude is above the high doorstep, it is made an edging. Major application of canny edge detector is for remote sensing images which are inherently noisy.

\section{IMPORTANCE OF CANNY DETECTION}

Despite of number of edge detection techniques available canny algorithm is careful because its contain a number of adjustable parameters which can affect the computation time and effectiveness of the algorithm.

a. The size of the Gaussian filter: The smoothing filter [18] used in the first step directly affects the results of the detection of small, sharp lines. A larger filter causes more blurring, smearing out the value of a given pixel over a larger area of image.

b. The use of two thresholds with hysteresis allows more suppleness than in a single threshold. A threshold set too high can miss important information. On the other hand, a threshold set too 
low will incorrectly recognize irrelevant information (such as noise) as important.

\section{CONCLUSION}

This paper presented a theoretical study of edge based image segmentation methods which provide insight into most widely used edge detection technique of Gradient-based and Laplacian base Edge Detection. This paper is to present a review of different approach for image segmentation based on edge detection techniques. The study of different Edge detection technique and their tentative grades shows that canny yield best results. In this paper an attempt is made to review the edge recognition technique which based on discontinuity intensity levels. The relative performance of various edge detection techniques are approved out with a picture by using MATLAB software. Different edge detection methods can be implement as per the require of segmentation of image .An adaptive edge-detection algorithm is necessary to pro-vide a robust explanation that is malleable to the varying noise level.

\section{REFERENCES}

[1] Santhosh Kumar, and J. Seetaram, "Medical Images Boundary Detection using a New Novel Algorithm and Gradient Features", International Journal of Engineering Research and Technology (IJERT), Vol. 1, Issue 8, pp. 14, October 2012.

[2] Jagadish H. Pujar, Pallavi S. Gurjal, Shambhavi D. S., and Kiran S. Kunnur, "Medical Image Segmentation based on Vigorous Smoothing and Edge Detection Ideology", International Journal of Electrical and Computing Engineering, Vol. 5, Issue 2, pp. 121-127, 2010.

[3] Dinesh D. Patil, and Sonal G. Deore, "Medical Image Segmentation: A Review", International Journal of Computer Science and Mobile Computing", Vol. 2, Issue 1, pp. 22-27, 1st Jan. 2013.

[4] Gautam A. Kudale, Mahesh Pawar, Joshi G.R., "Identification Of Annual Rings In Indeterminant Plants Using Different Edge Detection Methods", International Conference on Emerging Trends in Computer Science, Communication and Information Technology. Department of Computer Science \& Information, Technology,YeshwantMahavidyalaya, Nanded, Maharashtra, India. 9-11 January 2010.

[5] JagdishSangvikar, Gautam A. Kudale, Joshi G.R., "Noisy And Noiseless Image Compression Through Run Length Encoding Approach", A Three-day International Conference GIT- 2010 on "Green IT \& Open Source", (Approved by Ministry of HRD, Govt. of India), Sinhgad Institute of Management, Pune in association with University of Pune \& Computer Socity of India (CSI), (MS). India. 20-22 February 2010.

[6] Mohamed Roushdy, "Comparative Study of Edge Detection Algorithms Applying on the Grayscale Noisy Image Using Morphological Filter", GVIP Journal, Vol. 6, Issue 4, December, 2006.

[7] "Edge detection", (Trucco, Chapt 4AND Jain et al., Chapt 5).
[8] Wang Luo, "Comparison for Edge Detection of Colony Images", IJCSNS International Journal of Computer Science and Network Security, VOL.6 No.9A, September 2006.

[9] Brintha Therese1, Dr. S. Sundaravadivelu2, "Bipolar Incoherent Image Processing for Edge Detection of Medical Images", International Journal of Recent Trends in Engineering, Vol 2, No. 2, November 2009.

[10] Julian Guerrero, Septimiu E. Salcudean, James A. McEwen, Bassam A. Masri, and SavvakisNicolaou, "Real-Time Vessel Segmentation and Tracking for Ultrasound Imaging Applications", IEEE Transactions on Medical Imaging, Vol. 26, No. 8, pp. 1079-1090, August 2007.

[11] Francois Destrempes, Jean Meunier, Marie-France Giroux, Gilles Soulez, and Guy Cloutier, "Segmentation in Ultrasonic B-Mode Images of Healthy Carotid Arteries Using Mixtures of Nakagami Distributions and Stochastic Optimization", IEEE Transactions on Medical Imaging, Vol. 28, No. 2, pp. 215-229, February 2009.

[12] Juin-Der Lee, Hong-Ren Su, Philip E. Cheng, Michelle Liou, John A. D. Aston, Arthur C. Tsai and Cheng-Yu Chen, "MR Image Segmentation using a Power Transformation Approach", IEEE Transactions on Medical Imaging, Vol. 28, No. 6, pp. 894-905, June 2009.

[13] JiantaoPu, Joseph K. Leader, Bin Zheng, Friedrich Knollmann, Carl Fuhrman, Frank C. Sciurba, and David Gur, "A Computational Geometry Approach to Automated Pulmonary Fissure Segmentation in CT Examinations", IEEE Transactions on Medical Imaging, Vol. 28, No. 5, pp. 710-719, May 2009.

[14] S. Veeralakshmi, S. VanithaSivagami, V. Vimala Devi, and R. Udhaya, "Boundary Exposure using Intensity and Texture Gradient Features", IOSR Journal of Computer Engineering (IOSRJCE), Vol. 8, Issue 1, pp. 28-33, Nov.-Dec. 2012.

[15] K. Padmapriya, and T. K. Bino, "Boundary Detection using Edge Following Algorithm and Enhancement of the Image", International Conference on Computing and Control Engineering (ICCCE 2012), 12-13 April 2012.

[16] Sameer Antani, L. Rodney Long, George R. Thoma, D.J. Lee, "Anatomical Shape Representation in Spine X-ray Images".

[17] Guang CHEN,Keqin DING,Lihong LIANG, "A Method of weld Edge Extraction in the X-ray Linear Diode Arrays Real-time imaging", 17th World Conference on Nondestructive Testing, 25-28 Oct 2008.

[18] S. Das and A. Biswas and S. Dasgupta and A. Abraham, "Bacterial Foraging Optimization Algorithm: Theoretical Foundations, Analysis, and Applications", in Foundations of Computational Intelligence Volume 3: Global Optimization, pages 23-55, Springer, 2009. 